\title{
Replacement of Professional Photogrammetric Workstations with Low Cost or Free of Charge Photogrammetric Software and Services for Image Triangulation and Image Matching
}

\author{
Umesh Kumar \\ Survey Officer, Survey Department, Government of Nepal \\ Email: kumar24055@itc.nl,umesh2014@gmail.com
}

\section{Keywords}

Free Software, Image Triangulation, Image Matching, 123D Catch, PMVS, MATLAB, PMVS, RANSSE, PCM

\begin{abstract}
In the modern context of digital technology in the field of surveying and mapping, the technique of digital photogrammetry plays crucial role in all steps of mapping. There are some very expensive as well as low cost and free softwares are available in the market for professional photogrammetric work stations. The image triangulation and image matching is one of the very important steps of photogrammetry for which LPS as a commercial software is available in market. There are some low cost and free software as 123D Catch and Patch Boased Multi View Stereo Softwear (PMVS) also available. So, this paper mainly deals with the accuracy as well as the performance of those software for aerial triangulation and image matching for airborne image data to substitute commercial software for photogrammetric workstations. The accuracy assessment of image orientation, points cloud as well as DSM generated from those points cloud are also performed and compared with all products of LPS as commercial software.
\end{abstract}

\section{Introduction}

Photogrammetry is very important nowadays because it is the science of using 2D measurements to extract 3D information about position and geometry of the object (Goldbergs, 2012). There are many products of imagery as 3D city model, land use planning map, landscape map, environmental change application etc that directly or indirectly depend upon photogrammetry products and services (One, 2009). The importance of photogrammetry is also due to the fact that it provides updated digital data quickly for large area coverage.

Digital photogrammetry can be used with digital images as well as scanned photographs. It provides stereoscopic viewing with overlap photographs. It is possible to make 3D measurements very precisely from it. From the few decades' history of photogrammetry, it can be seen that the development of computer technology also advanced the photogrammetry in the area of instrumentation, methodology and integration. Current mapping system of photogrammetry is based on personal computer and workstation environment (Madani, 2001).

Image triangulation provides the exterior orientation parameters of two or more overlapping images. Image matching establishes correspondence between pixels or feature points in two overlapping images. Image orientation and image matching process and production of $3 \mathrm{D}$ information is very important to demonstrate and to solve many real world problems.

In production of objects information from image, there is need of reliable software. Some of that 
software has fully automated functionalities and some are semi-automated. So it needs availability of free or low cost software with support for automated methods to keep costs within reasonable bounds. The software should seek to provide not only this object's information but also provide quality results in semiautomatic or fully automated algorithms. There are different types of softwares packages available in the market which does not contain information on how accurate they are in image triangulation and image matching object measurements to guarantee their reliability and possible comparison with the conventional in most cases cost prohibitive software packages.

Further work needs to be done to see how accurate these free/low cost software are in image triangulation and image matching as well as 3D topographical objects measurements. There are many software available in the market either they are free or low cost or fully commercial. Their performance and accuracy are also different to each other. The Autodesk product 123D Catch (Autodesk, 2012), and PMVS (Furukawa, 2010) software are also available free and low cost. 123D catch software is used for image matching in local coordinates system it computes the 3D scene. PMVS is used for dense point cloud production. This paper tries to test their performance in comparison with fully commercial software as ERDAS LPS. These free softwares are user friendly and also support all familiar operating systems which need for image triangulation and image matching.

\section{Image Orientation}

The image orientation determines the mathematical relation between the object (terrain) and the image coordinates. The main task of image orientation is to reconstruct the geometric relations between an object point $(\mathrm{U}, \mathrm{V}, \mathrm{W})$ and its image (X', Y') co-ordinates by calculations based on the collinearity equations. Aerial triangulation is a process of assigning ground coordinates to a sufficient number of image points in the photogrammetric models for a large number of photographs. The aim of aerial triangulation is to determine the parameters of exterior orientation (and additional parameters by self calibration) of a block of images and simultaneously the object coordinates of the points tie, check and control points (Goldbergs, 2012).

This paper tries to demonstrate four major steps for addressing the problem. The tasks are data preparation both image data and ground control points data, image orientation and image matching from both free or low cost software and LPS software, accuracy assessment, DSM creation and comparison.

\section{Workflow}

Resampled images are uploaded into 123D Catch software. It provides web services for computing 3D scene and provides automatic image matching in local coordinate system. The oriented image in 123D Catch as a scene is exported in .rzi file. The exported .rzi file is used for separate bundle block adjustment in PMVS software for dense point production. In parallel the image orientation is done with original images in LPS software and dense point cloud production is also performed. Some locators has already measured in 123D Catch software to determine the transformation parameters as well as check the accuracy of image orientation and matching. The corresponding global coordinates of locators are measured in LPS project. The point clouds obtained in local coordinates system from 123D Catch, PMVS are transformed into global coordinate system using code written in MATLAB software. Finally, DSM is created from all three sets of points clouds and accuracy assessment is performed.

The local coordinates are measurement indices into a local coordinate space. It is useful for their ability to model independently transformable aspects of geometrical scenegraphs (Wikipedia, 2012b). The global coordinate system is constructed to match the surface of the earth (Commission, 2012). 
The workflow diagram for all process is as follows:

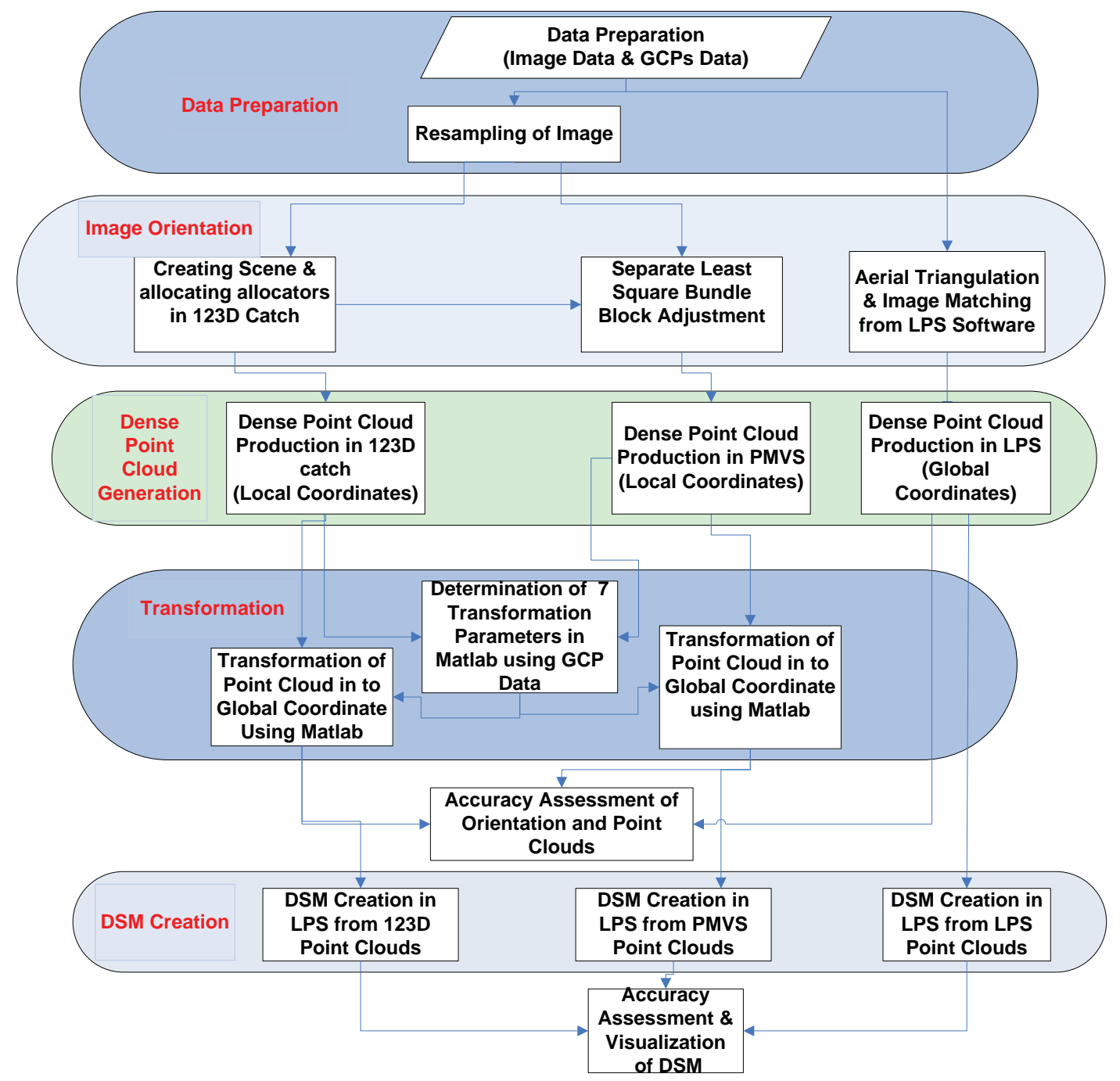

Figure 1: Applied methodology

\section{Image Orientation Using 123D Catch Software}

The resampled and fiducial marks cropped image data are used for orientation in 123D catch software. The next step is the orientation of the images and creation of the true 3D model of images. For, this the sequence of images in the same sequence of capturing is uploaded into $123 \mathrm{D}$ catch software.

This software provides web services so the resampling of images is necessary to reduce the volumes of images for convenience to upload and downloading. After a while it provides 3D scene ready, in which orientation is done by software without announcing the procedure. But normally it uses the "Structure From Motion" (SFM) algorithm (Snavely, 2010), in which the 3D structure is extracted through the two consequence images, considering the correspondence points in images and extracting the camera geometry or corresponding geometry of images (epipolar geometry). The corresponding points are founded by SIFT algorithm. In this procedure, the scale and rotation and illumination deformation of camera and picture capturing devise are not necessarily stable. But as we know, in this method so many wrong points are selected, so we use RANSAC algorithm to detect and eliminate the wrong correspond points. 
There are other algorithms than SFM, to calculate the orientation parameters through the correspondent points. The outputs of 3D scene from 123D Catch software with position of aerial camera and locators are shown below:

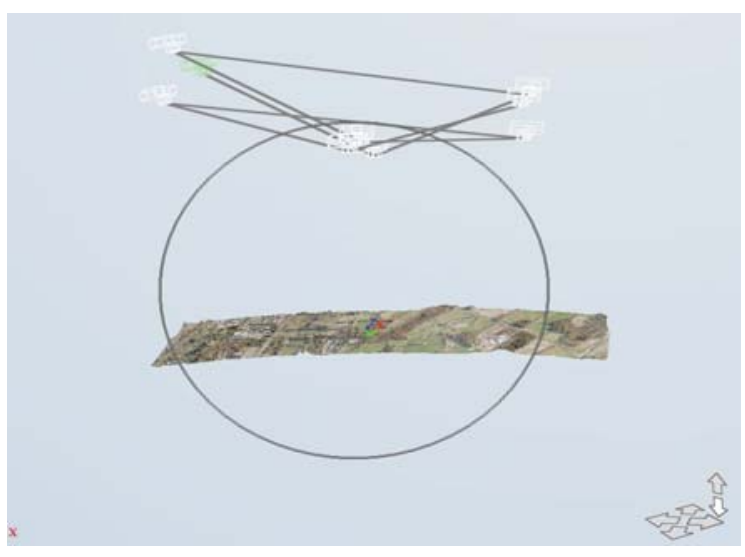

a

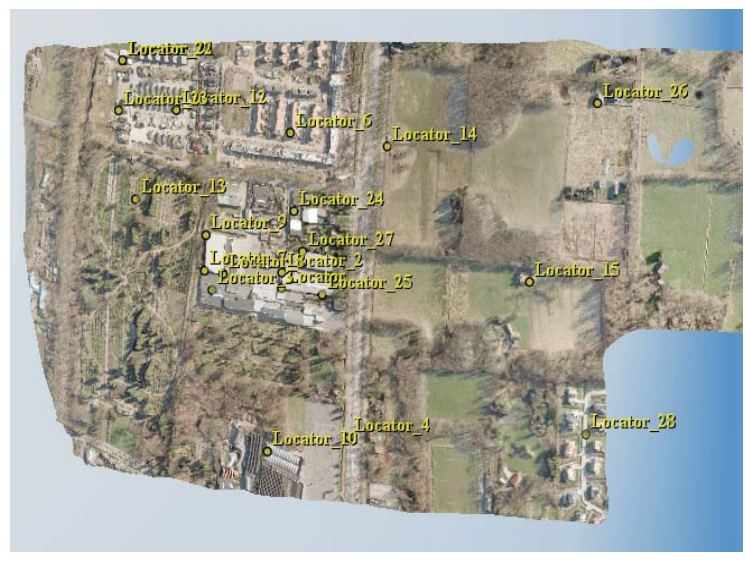

b

Figure 3: 3D scene of Enschede, The Netherlands image with camera position in (a) and locators in (b)

After computing the 3D scene in 123D Catch some well defined reference points are selected and best matches of them in several images are found. Locators (it is three dimensional point stored in the computer and equivalent to tie point) are allocated for this reference points. Local coordinates of this reference points can be determined. Then the models created in $123 \mathrm{D}$ catch with locators are exported as .rzi file.

The global coordinates of these corresponding points of locators are determined very precisely manually from LPS software in point measurements tools of already created aerial triangulation model.

From the Fig 3a it can be seen that the 3D scene created by $123 \mathrm{D}$ Catch software make the surface curve as convex upward. This is due to the image orientation done in this software without ground control points(GCP). There is not provision to perform image orientation in 123D Catch software with GCP. This is due to on availability of height information with ground control points. Figure $3 \mathrm{~b}$ shows the measured position of locators in $3 \mathrm{D}$ scene of Enschede, The Netherlands image data.

\section{Separate Bundle Adjustment by Least Square Adjustment}

Separate bundle block least square adjustment is performed to use image orientation in PMVS software format.

\section{Dense Point Matching Using PMVS Software}

The other method which is different from the global matching methods is PMVS. In this method, the only search around the seed point and in each step it enlarges the search area. The patch matching optimization is usually done through least square algorithm. The point cloud is generated in PMVS software.

\section{DSM Generation in LPS Software}

The point cloud obtained from 123D Catch, PMVS and LPS software are used for DSM generation. The point cloud generated in LPS is $1 \mathrm{~m} * 1 \mathrm{~m}$ resolution, standard methods are used to generate point cloud in 123D Catch and default settings are used in PMVS to generate point clouds. The density of point cloud of 123D Catch is very low so it is not used for DSM generation and comparison. The DSM is created in LPS software from LPS and PMVS point clouds.

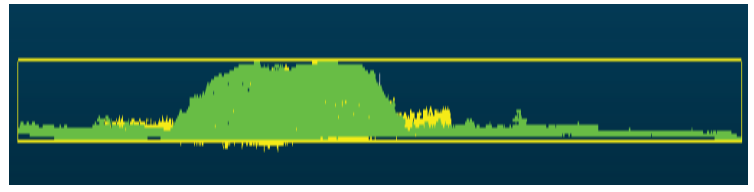

Figure 4: Display of both LPS and PMVS point clouds in point cloud compare software 


\section{Quality Assessment of Image Orientation and Image Matching}

After computing the 3D scene in 123D Catch, some reference points are selected and the best matches of them in several images as locators are found. In LPS, it can be measured some ground control points to calculate the absolute orientation parameters. Then the model is exported as rzi file and converted into .txt file containing only point's coordinates in local system. It can be used for transformation to get global coordinate system.

The transformation parameters can be determined from local coordinates of manually measured locators and corresponding global coordinates measured in LPS project. To perform transformation the code written in Matrix Laboratory (MATLAB) can be used. There are 3 elements of rotation matrix and 3 translation vector and 1 scale factor (all together 7 parameters). To calculate 7 transformation parameters at least 3 measured points are used and other points can be used for accuracy assessment. Then through these transformation parameters it can calculated the transformed global coordinates of all points cloud. This residuals values show how accurate was this model made, because it has used the measured points on the $3 \mathrm{D}$ model and compared
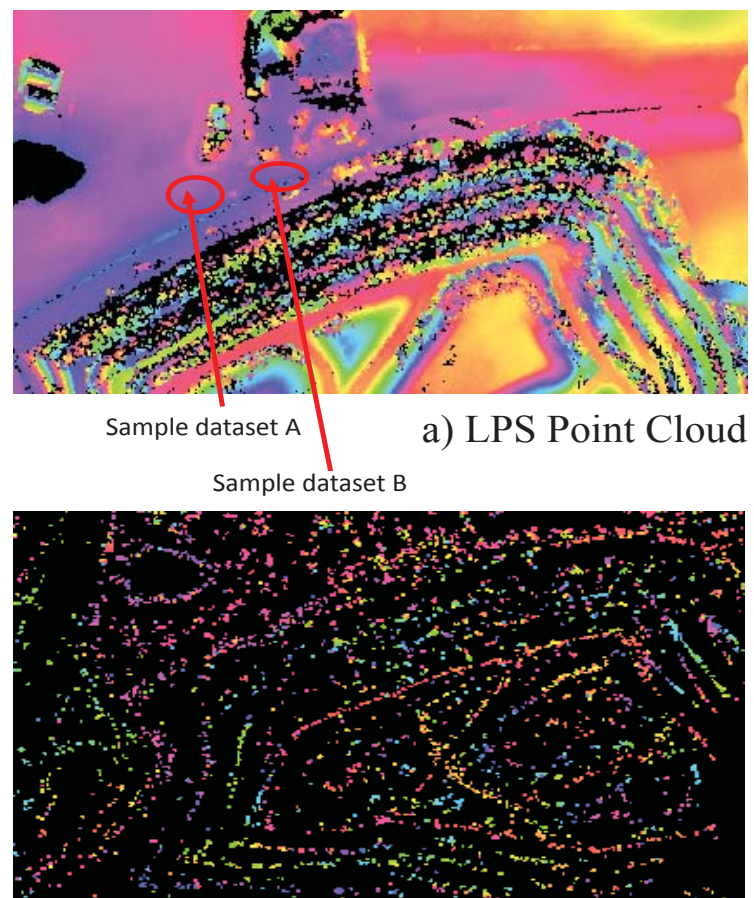

c) 123D Catch Mobile Point Cloud them to the measurements by LPS points coordinate. At the end, there are transformed coordinates for all point clouds as from 123D Catch, PMVS, which can be used for other analysis.

\section{Quality Assessment of Points Clouds}

The main task is to access of the quality of the orientation of the point clouds. Several quality aspects are considered for individual points, point density, gaps or occlusion etc. There are three options in 123D Catch to produce point cloud:

- Mobile: This is suitable for medium resolution mesh. It is fast and suitable for viewing on mobile devices.

- Standard: It is recommended for high resolution textured mesh and used to visualization on the desktop computer.

- Maximum: It is used for very high density mesh. The output may be maximum quality but it takes long processing time.

The mobile and standard method is chosen for output quality to produce point cloud in this project. The mobile as well as standard method provides almost same quality as well as quantity of point clouds for the same resolution of image data. For the following points cloud colour cycle length is $10 \mathrm{~m}$ i.e. the sequential difference of adjacent colour is $10 \mathrm{~m}$.

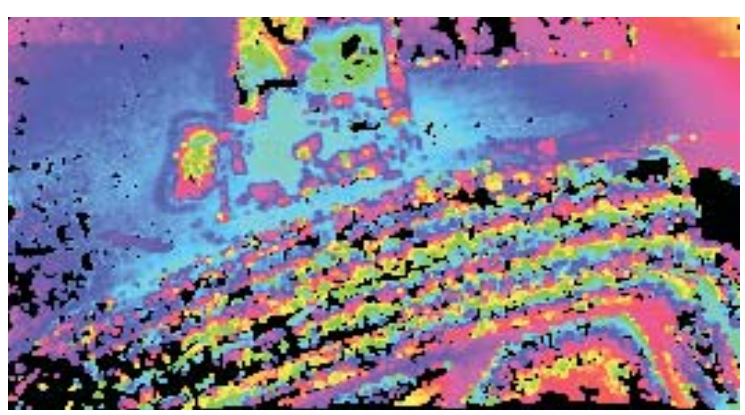

b) PMVS Point Cloud

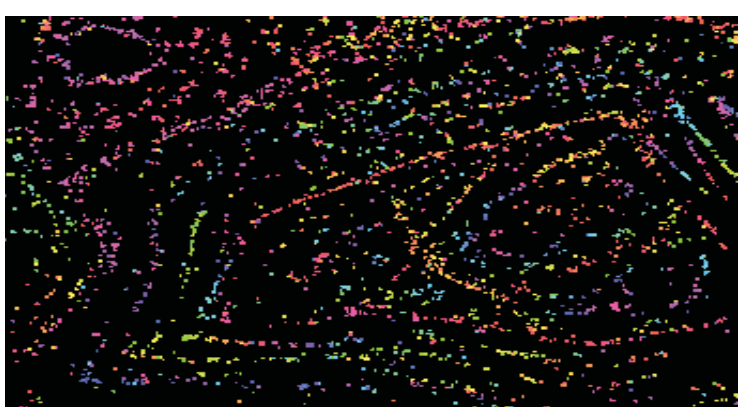

d) 123D Catch Standard Point Cloud

Figure 5: Visualization of Points Cloud of Moers, Germany data in PCM software 
It has been observed that LPS can produce more dense point cloud than PMVS as well as 123D Catch. The Figure 5(a) shows point cloud generated in LPS by $1 \mathrm{~m}^{\star} 1 \mathrm{~m}$ resolution settings. The point density of $123 \mathrm{D}$ Catch either created from both methods as mobile and standard are almost same and low compared to PMVS as well as LPS eATE point cloud. Point's cloud of LPS mostly represents ground surface but PMVS point cloud contains features as buildings, trees etc.

The principal component analysis (PCA) is applied to determine plane parameters. The principal components are the axis of maximum variation of the data. The robust fitting algorithm gives inliers which are used to obtain the point to the plane distances. The Random Sample Consensus (RANSAC) algorithm is used to perform robust plane fitting. The mean and standard deviation of the point-to-plane distances after the plane fitting were computed. The mean of point plane distances is expected to be very small. The standard deviation indicates the random errors of the point (Sande, Soudarissanane, \& Khosheham, 2010). The quality of point cloud can be performed by two different perspectives as internal and external quality. In external quality assessment it can be considered using corresponding lines and corresponding planes to make comparison with the LPS point dataset which is considered as higher accuracy. For plane based accuracy assessment, it can be used the algorithm proposed by Dr. Kourosh (Sande et al., 2010). The two sets of point clouds datasets are imported into Point Cloud Mapper (PCM) software and two groups of corresponding planes are selected into two interfaces. Then these are imported into the Matlab software and used to fit point to plane algorithm to make the points in the selected LPS plane to one plane, then the distance between this plane with the planes of PMVS and 123D Catch plane are calculated.

In internal quality assessment one plane from the point cloud generated by PMVS or 123D Catch or LPS are selected and it is used to fit plane to point algorithm to fit the selected point to one flat plane. Then the distance from the original point plane with the plane after processing are computed. The codes used in Matlab software for internal accuracy assessment and external accuracy assessment.

The samples data from corresponding planes are cropped in PCM software and imported into Matlab software code and processing is performed. The different statistical analysis (Mean, SD, Minimum Distance, Maximum Distance, Median Distance, 25th percentile and 75th percentile) are also computed to perform this accuracy assessment. Different graphs are also plotted for comparison of each dataset. For this statistical comparison different sample data sets are taken from different areas. One sample dataset is taken from relatively plane area and other is taken in slope area.

\section{Conclusion}

The two datasets as one taken from analogue aerial camera and another from digital aerial camera with different specification are used for study. The images are also used with different resolutions and different area types as mountainous and flat terrain. There are different types of accuracy assessment perform to check the orientation of images, generated point cloud and created DSM from point cloud obtained from 123D Catch, PMVS and LPS software.

123D Catch software provides web services for orientation of image and create 3D scene in local coordinate system. There are three options in 123D Catch to produce point clouds are mobile, standard and maximum. The mobile and standard method is chosen for output quality to produce point cloud in this project. The mobile as well as standard method provides almost same quality as well as quantity of point clouds for the same resolution of image data. The maximum methods give some more dense point cloud compare to mobile and standard methods. The standard methods take long processing time to generate $3 \mathrm{D}$ scene.

For image orientation accuracy, it has been observed that for large area the orientation in 123D Catch software makes model as curved surface, this is due to not provision to use ground control point with height information during orientation in this software. So, this software is not suitable for large area and for long strip aerial data. The orientation accuracy is high for continuous rugged surface than plane with high settlement features. It can be observed that the accuracy obtained for planimetric is more accurate than altimetric in image orientation due to curved $3 \mathrm{D}$ scene created by $123 \mathrm{D}$ Catch software. It has been observed that the well distributed locators in the study area provide good transformation parameters. 123D Catch software not needs intensive training for their operation for image triangulation and image matching as like LPS software.

The 3D model created by 123D Catch software is exported into .rzi file. It contains local coordinates system of point clouds. This .rzi file is also used for separate bundle block adjustment to generate point clouds in PMVS software. This local coordinates point clouds are transformed into global coordinates. LPS as commercial software has also facilities to generate point clouds in required density. The accuracy assessment of 123D Catch points cloud and PMVS point cloud is done compare to LPS point clouds because it has used high resolution image for orientation and considered as more accurate points cloud.

PMVS bundle block adjustment provides high quality of point cloud and relatively more dense point by using .rzi file created from 123D Catch software. 123D Catch point cloud contains more systematic and random errors comparison to PMVS points clouds 
because the value of mean and standard deviation are high. The DSM created by point cloud of PMVS seems to be more reliable than 123D Catch and LPS. The DSM created from PMVS points cloud contains high pixel values than the DSM of LPS points cloud because PMVS point clouds mostly contains features like buildings, trees etc.

\section{References}

Autodesk. (2012). Autodesk 123D Catch. Autodesk 123D Catch Retrieved 22 June 2012, 2012, from http://www.123dapp.com/catch

Commission, U. C. o. F. (2012). US Centennial of Flight Commission Retrieved 19 August 2012, 2012, from http://www.centennialofflight.gov/essay/ Dictionary/Global_Coordinates/DI80.htm

Ebadi, H. (2006). Advanced Analytical Aerial Triangulation: K.N.TOOSI UNVERSITY.

Fritsch, D. (1995). Introduction into Digital Aerotriangulation Photogrammetric Week '95'.

Furukawa, Y. (2010, 13 July 2010). Patch-based Multiview Stereo Software Retrieved 22 June 2012, 2012, from http://grail.cs.washington.edu/software/pmvs/

Bhatta, G.(2009)Applications of open source software in land administration: An initiation with land administration education, Nepalese Journal on Geoinformatics, Volume 8

Gerke, M. (2012). Base Mapping From Images. Module 7, GFM Master Lecture Notes.

Girardeau, D. (2012). 3D point cloud and mesh processing software Retrieved 14 August 2012, 2012, from http://www.danielgm.net/cc/

Goldbergs, G. (2012). Image Triangulation and Orientation. Module 5, GFM Master Lecture Notes.

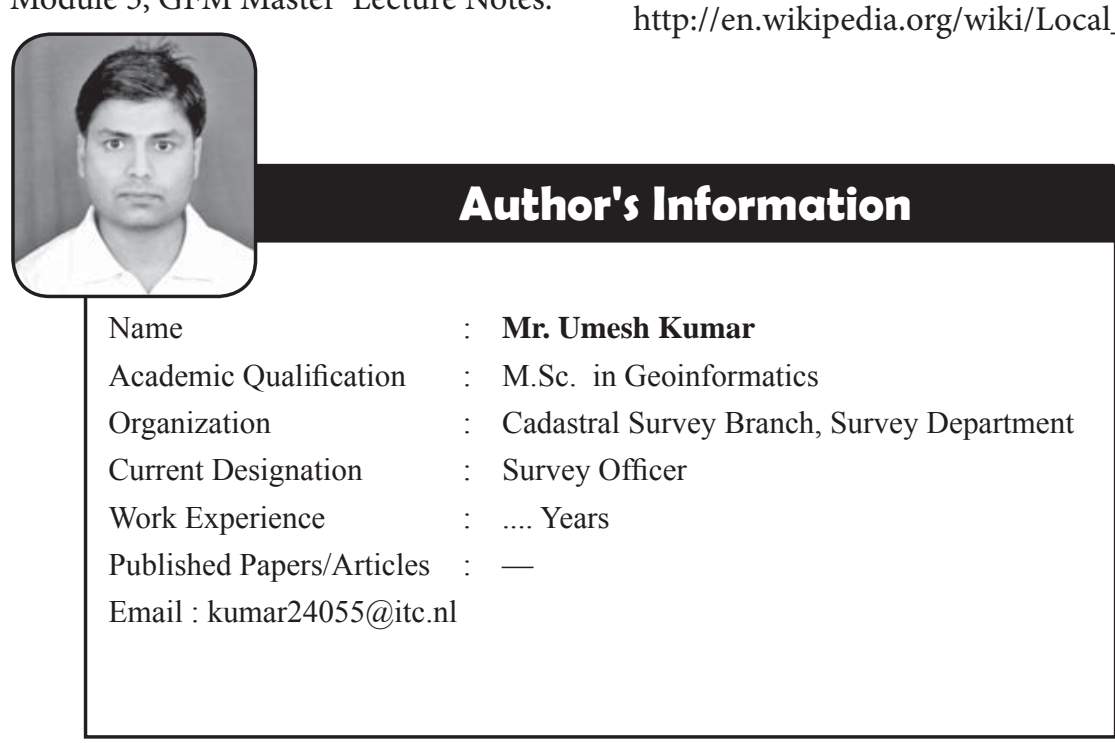

Google. (2012). Google Maps Retrieved 5 August 2012, 2012, from https://maps.google.com/ maps?hl=en

Khoshelham, K. (2012). Quality of Terrestrial Point Clouds Module 13, M.Sc. Lecture Notes.

Madani, M. (2001). Importance of Digital Photogrammetry for a complete GIS. Paper presented at the 5th Global Spatial Data Infrastructure Conference, Cartagena, Columbia.

One, V.(2009, 11 September 2009). Is Photogrammetry Important to the Geospatial Industry? Retrieved 16 August 2012, 2012, from http://www.sensysmag. com/vectorone/?p=3701

Sande, C. V., Soudarissanane, S., \& Khosheham, K. (2010). Assessment of Relative Accuracy of AHN-2 Laser Scanning Data Using Planar Features. Retrieved from sensor website: http://www.mdpi.com/14248220/10/9/8198/htm

Snavely, N. (2010). Bundler: Structure from Motion (SfM) for Unordered Image Collections Retrieved 12 July 2012, 2012, from http://phototour.cs.washington. edu/bundler/

Suite, L. P. (2006). Leica Photogrammetry Suite Automatic Terrain Extraction. United States: Leica Geosystems Geospatial Imaging.

Tempfli, K. (2008). Digital Surface Model. GFM Master Course, ITC The Netherlands.

Wikipedia. (2012a, 14 August 2012 ). Box Plot Retrieved 15 August 2012, 2012, from http:// en.wikipedia.org/wiki/Box_plot

Wikipedia. (2012b, 16 December 2009). Local Coordinates Retrieved 19 August 2012, 2012, from http://en.wikipedia.org/wiki/Local_coordinates 